\title{
Vitamin A supplementation and childhood morbidity from diarrhea, fever, respiratory problems and anemia in sub-Saharan Africa
}

\author{
Samson Gebremedhin \\ School of Public and Environmental \\ Health, Hawassa University, Hawassa, \\ Ethiopia
}

This article was published in the following Dove Press journal:

Nutrition and Dietary Supplements

28 July 2017

Number of times this article has been viewed

Background: Systematic reviews consistently indicated that vitamin A supplementation (VAS) (100,000-200,000 international unit [IU] semiannually) to children aged 6-59 months substantially reduces all-cause child mortality. Yet, its effect on morbidity is inconsistent and its contribution to the reduction of anemia has not been sufficiently explored.

Objective: The objective of this study was to assess the association between routine VAS (100,000 IU for children aged 6-11 months and 200,000 IU for children aged 1-5 years) and the occurrence of common childhood illnesses (fever, diarrhea, acute respiratory infection [ARI] and anemia) in preschool children in sub-Saharan Africa (SSA).

Methods: The analysis was made based on the data of 28 demographic and health surveys (DHSs) conducted in SSA since 2010. The data of 152,406 children were included. The VAS status in the preceding 6 months and the occurrence of the illnesses in the past 2 weeks were determined based on the information given by the caregivers. The hemoglobin level was determined using a portable photometer. Data were analyzed using mixed-effects logistic regression model. The outputs were provided using adjusted odds ratio (AOR) with the respective 95\% confidence intervals (CIs). The practical significance of the association was measured via Cohen's effect size $(d)$.

Results: The coverage of VAS was 56.3\% (95\% CI: 56.1-56.5). VAS was modestly associated with increased odds of fever ( $\mathrm{AOR}=1.12$ [95\% CI: 1.09-1.15]), diarrhea (AOR=1.09 [95\% CI: 1.05-1.13]) and ARI (AOR=1.18 [95\%: 1.12-1.24]). Conversely, in the supplemented children, the odds of anemia were reduced by $10.1 \%$ (95\% CI: $7.0-13.1)$. All the associations translated into trivial effect sizes $(d<0.2)$.

Conclusion: VAS is associated with an inconsequential increase in the occurrence of common childhood ailments.

Keywords: vitamin A supplementation, diarrhea, fever, acute respiratory infection, anemia, hemoglobin, demographic and health survey, sub-Saharan Africa

\section{Background}

Vitamin A plays a vital role in a range of physiological functions, including vision, immunity, growth, hematopoiesis and reproduction. ${ }^{1}$ The significance of the nutrient for the innate and adaptive immune functions has been extensively studied. ${ }^{2}$ Vitamin A deficiency (VAD) - serum retinol level $<0.70 \mu \mathrm{mol} / \mathrm{L}$ - impairs integrity of the mucosal epithelium of the gut, diminishes the functions of phagocytic and natural killer cells and alters the functions of T- and B-cells. ${ }^{2,3}$

Globally, it was estimated that 190 million preschool children are vitamin A deficient. ${ }^{4}$ Sub-Saharan Africa (SSA) and Southeast Asia are the most affected regions.
Correspondence: Samson Gebremedhin School of Public and Environmental Health, Hawassa University, PO Box 12485, Addis Ababa, Ethiopia

Tel +25I 9 I682 28I5

Email samsongmgs@yahoo.com 
In $\mathrm{SSA}, 56$ million children, $44.4 \%$ of all preschoolers, are Vitamin A deficient. ${ }^{4}$ In 44 of the 49 countries in the $\mathrm{SSA}$ region, VAD has severe degree of public health significance. ${ }^{4}$ According to the United Nations Children's Fund (UNICEF), in 2013, 73\% of children aged 6-59 months in the SSA region received two doses of vitamin A supplementation (VAS). ${ }^{5}$

Meta-analyses of randomized controlled trials (RCTs) from developing countries availed persuasive evidence that VAS to children aged 6-59 months (100,000 international unit [IU] for children aged 6-11 months and 200,000 IU for children aged 1-5 years) reduces all-cause mortality by 23-30\%. ${ }^{6-10}$ It also cuts diarrhea-specific mortality but not deaths secondary to measles, meningitis and pneumonia. ${ }^{9} \mathrm{~A}$ recent review concluded, given the existing unequivocal evidence, that there is no need for additional placebo-controlled VAS trials in children aged 6-59 months. ${ }^{7}$

Nonetheless, the association between VAS and incidence of common childhood morbidities, including diarrhea and acute respiratory infections (ARI), has not been consistent. ${ }^{10,11}$ A recent meta-analysis concluded that VAS significantly reduced the incidence of diarrhea by $15 \% ;^{9}$ however, earlier reviews reported no association between them. ${ }^{6}$ Furthermore, systematic reviews found no association between VAS and the incidence of ARI, ${ }^{6,10}$ and even a couple of meta-analyses indicated that VAS may slightly increase the risk of ARI. ${ }^{12,13}$

VAD is regarded as a cause of nutritional anemia. ${ }^{14}$ Although the mechanism whereby vitamin A affects hemoglobin status has not been fully elucidated, ${ }^{15}$ it appears to be involved in hematopoiesis and enhances mobilization of iron stores. ${ }^{14,16,17}$ It could also prevent anemia of infection via its immune-enhancing role. ${ }^{14,17}$ However, empirical evidence on the actual public health impact of VAS for anemia is scarce. ${ }^{14}$

The purpose of this analysis was to assess the association between VAS and morbidity from diarrhea, fever, ARI and anemia among children aged 6-59 months, based on the data of 28 recent national demographic and health surveys (DHSs) conducted in SSA.

\section{Methods}

\section{Study setting}

The SSA includes 49 predominately low-income African countries located south of the Sahara. ${ }^{18}$ According to the World Health Organization (WHO), based on the prevalence of low serum retinol level in preschool children, in 44 of the 49 SSA countries, VAD has a severe degree of public health significance. ${ }^{4}$ Similarly, in 25 of the 28 countries included in this analysis, - excluding Rwanda, Namibia and Gabon - VAD had severe public health significance. ${ }^{4}$ In settings where VAD is a public health problem, high-dose semiannual and routine VAS (100,000 IU for children aged 6-11 months and 200,000 IU for children aged 1-5 years) is recommended. ${ }^{19,20}$ Accordingly, SSA countries have integrated VAS program into their child survival interventions, and a significant progress in coverage has been achieved over the past decade. ${ }^{21}$

\section{Study design}

Secondary data analysis was based on 28 DHSs conducted in SSA since 2010.

\section{Sample size and representation of countries}

The data of 152,406 children aged 6-59 months having completed information regarding the VAS status and age were included in the analysis. The distribution across the countries is summarized in Table 1.

Table I List of 28 surveys included in the analysis, SSA, 20102014

\begin{tabular}{lll}
\hline Country & $\begin{array}{l}\text { Year of data } \\
\text { collection }\end{array}$ & $\begin{array}{l}\text { Sample } \\
\text { size }\end{array}$ \\
\hline Benin & $2011 / 2012$ & 10,567 \\
Burkina Faso & 2010 & 6,047 \\
Burundi & 2010 & 3,236 \\
Cameroon & 2011 & 3,560 \\
Comoros & 2012 & 2,428 \\
Republic of Congo & $2011-2012$ & 4,077 \\
Democratic Republic of Congo & $2013-2014$ & 7,427 \\
Côte d'lvoire & $2011-2012$ & 3,007 \\
Ethiopia & 2011 & 9,166 \\
Gabon & 2012 & 3,045 \\
The Gambia & 2013 & 3,136 \\
Ghana & 2014 & 2,435 \\
Guinea & 2012 & 2,832 \\
Liberia & 2013 & 2,958 \\
Malawi & 2010 & 4,648 \\
Mali & $2012-2013$ & 4,312 \\
Mozambique & 2011 & 8,729 \\
Namibia & 2013 & 1,633 \\
Niger & 2012 & 4,817 \\
Nigeria & 2013 & 24,332 \\
Rwanda & 2010 & 3,761 \\
Senegal & 2014 & 5,559 \\
Sierra Leone & 2013 & 4,313 \\
Tanzania & 2010 & 6,331 \\
Togo & $2013-2014$ & 2,965 \\
Uganda & 2011 & 1,930 \\
Zambia & $2013-2014$ & 11,051 \\
Zimbabwe & $2010-2014$ & 4,104 \\
\hline Abbreviatis & \\
\hline
\end{tabular}

Abbreviation: SSA, sub-Saharan Africa. 


\section{Sampling approach of the DHS}

The DHS was designed to generate representative data at national, place of residence (urban and rural) and regional (states) levels. The sampling was based on a stratified twostage cluster design. Initially, the country was stratified based on region and place of residence. Then, from each stratum, a predetermined number of enumeration areas (EAs) defined during the most recent national population census were selected using the probability proportional to size (PPS) approach. In each selected EA, a sample of households (usually 20-25) was randomly drawn from an updated sampling frame. All eligible children in the selected households were included in the study. ${ }^{22}$

\section{Data collection approach of the DHS}

DHS data were gathered by trained interviewers and supervisors using standardized questionnaires prepared in the major local languages. Information including VAS status of the index child in the preceding 6 months and history of illness (cough, fast breathing, fever and diarrhea) in the last 2 weeks was collected from the mothers/primary caregivers of the children. ${ }^{22}$ Children with both cough and fast breathing were considered as having ARI. Across the 28 surveys, VAS status and the occurrence of childhood illness were determined solely based on the response of the caregivers without any clinical or laboratory investigations.

The hemoglobin concentration was determined using a portable $\mathrm{HemoCue}{ }^{\circledR}$ photometer using capillary blood samples. ${ }^{22}$ Hemoglobin was adjusted for altitude according to the recommendation of International Nutritional Anemia Consultative Group (INACG) ${ }^{23}$ Anemia was defined as hemoglobin concentration lower than $11 \mathrm{~g} / \mathrm{dL} .^{23}$ The variables included in the current analysis were measured similarly across the 28 surveys.

\section{Data management and analysis}

The datasets of all standard DHS surveys conducted in the SSA since 2010 were separately downloaded from the Monitoring and Evaluation to Assess and Use Results Demographic and Health Surveys (MEASURE DHS) website and merged into a mega file. Analysis was made using STATA SE 12 software. Data of children $<6$ months of age and observations that missed key variables (child age and VAS status) were excluded. Descriptive statistics were computed using the weight analysis approach. The analytic weight was defined based on the sampling weight readily available in the datasets and the post-stratification weight developed based on the 2013 population size of the countries.
The coverage of VAS was computed as a proportion of children who reportedly received oral vitamin A supplement in the previous 6 months of the survey, out of the total children aged 6-59 months. For each of the 28 countries represented, the coverage of VAS was presented with the respective 95\% confidence intervals (CIs). The coverage of VAS was compared across categories of child's age, maternal education, place of residence (urban or rural) and household wealth index using multivariate logistic regression analysis.

The association between VAS and childhood illnesses was determined by a mixed-effects logistic regression analysis. The outputs of the model were presented using crude odds ratio (COR) and adjusted odds ratio (AOR). The models were adjusted for potential confounders, namely, child age, maternal educational status, wealth index (a composite measure of a household's living standard developed based on ownership of selected assets), place of residence, number of children younger than 5 years in the household, vaccination status and type of household's drinking water source. $P$-value of 0.05 was taken as the level of significance.

Studies based on large sample size are likely to detect small statistical differences that are not practically relevant. ${ }^{24}$ Accordingly, in additional to the traditional $P$-value, effect size - the net magnitude of the difference between groups should be presented. ${ }^{25}$ In the current study, the practical significance of the observed associations was evaluated by converting AOR values into Cohen's effect sizes $(d)$ using an online program. ${ }^{26}$ Effect size $<0.2$ was considered as trivial effect. ${ }^{27}$

Household water sources and sanitation facilities were classified as improved and non-improved according to the definition of the WHO/UNICEF. ${ }^{28}$ Regarding water sources, piped water into dwelling or to yard, public standpipe, borehole, protected well, protected spring and rain water were considered as improved sources, whereas others were taken as non-improved sources. ${ }^{28}$ Regarding sanitation facilities, flush toilet, toilet connected to sewer system, septic tank, ventilated improved pit latrine, traditional pit latrine with slab and composting toilet were considered as improved facilities. All others, including shared facility of any type, were considered as non-improved facilities. ${ }^{28}$

Anthropometric indices were determined based on the WHO growth reference. Stunting, underweight and wasting were defined based on the standardized height-for-age, weight-for-age and weight-for-height scores, respectively. 


\section{Ethical considerations}

The datasets were accessed after securing permission from the DHS Program. ${ }^{29}$ The primary data were collected in accordance with international ethical guidelines. For each survey, ethical clearances were secured from the institutional review board (IRB) of ORC Macro and national level IRBs of all host countries. Data were collected after taking written informed consent from mothers/care takers of the index children.

\section{Results}

\section{Characteristics of the study children}

The data of 152,406 children were included in the analysis. The male-to-female ratio was 1.02 and 12.6 , and $87.4 \%$ of the children were between 6-11 and 12-59 months of age. The mean age $( \pm \mathrm{SD})$ of the respondents was 29.4 years ( \pm 6.8 years), and $46.3 \%$ had no formal education. A quarter $(27.9 \%)$ of the respondents was sampled from rural areas. More than one-third of the children lived in households that used unimproved drinking water sources (40.6\%) and sanitation facilities $(37.6 \%)$. The prevalence of stunting, underweight and wasting in the index children was 36.0, $30.3,10.4 \%$, respectively (Table 2 ).

\section{Coverage of VAS in children aged 6-59 months}

Among children 6-59 months, the coverage of VAS was $56.3 \%$ (95\% CI: 56.1-56.5). Among the 28 countries represented, seven countries (Rwanda, Senegal, Malawi, Namibia, Sierra Leone, Togo and Burundi) achieved $80 \%$ or higher coverage; conversely, four countries (Guinea, Nigeria, Comoros and Benin) had $<50 \%$ coverage (Figure 1 ).

The coverage of VAS appeared to vary across categories of children's age and socioeconomic status. Children aged 6-11 months were less likely to have received VAS (48.6\%) than older children $(57.9 \%)$. The coverage in urban areas $(63.9 \%)$ was significantly higher than that of rural areas $(53.9 \%)$. Better maternal education and household wealth index were associated with higher utilization of VAS (Table 3 ).

\section{VAS and diarrhea, fever and ARI symptoms}

Approximately 19.0, 14.4 and 5.1\% of the children had fever, diarrhea and ARI in the preceding 2 weeks of the surveys. The prevalence of the three ailments was slightly higher in supplemented children compared to their counterparts $(p<0.05)$. Similarly, in the multivariate logistic model, the odds of fever (1.12 [95\% CI: 1.09-1.15]), diarrhea (1.09 [95\% CI: 1.05-1.13]) and ARI (1.18 [95\% CI: 1.12-1.24])
Table 2 Characteristics of the study children, 28 SSA countries, 2010-2014

\begin{tabular}{|c|c|}
\hline Variables & Percentage \\
\hline \multicolumn{2}{|l|}{$\operatorname{Sex}(n=152,406)$} \\
\hline Male & 50.4 \\
\hline Female & 49.6 \\
\hline \multicolumn{2}{|c|}{ Age in months $(n=152,406)$} \\
\hline $6-11$ & 12.6 \\
\hline $12-23$ & 23.0 \\
\hline $24-35$ & 21.5 \\
\hline $35-47$ & 22.1 \\
\hline $48-59$ & 20.7 \\
\hline \multicolumn{2}{|c|}{ Place of residence $(n=\mid 52,406)$} \\
\hline Urban & 27.9 \\
\hline Rural & 72.1 \\
\hline \multicolumn{2}{|c|}{ Educational status of the respondents $(n=\mid 52,387)$} \\
\hline No formal education & 46.3 \\
\hline Primary education & 32.1 \\
\hline Secondary education & 18.7 \\
\hline Tertiary education & 2.9 \\
\hline \multicolumn{2}{|c|}{ Type of drinking water source $(n=150,269)$} \\
\hline Improved & 59.4 \\
\hline Non-improved & 40.6 \\
\hline \multicolumn{2}{|c|}{ Type of household sanitation facility $(n=|50,3| 5)$} \\
\hline Improved & 62.4 \\
\hline Non-improved & 37.6 \\
\hline \multicolumn{2}{|c|}{ Stunting status $(n=\mid 38,757)$} \\
\hline Stunted & 36.0 \\
\hline Not stunted & 64.0 \\
\hline \multicolumn{2}{|c|}{ Underweight status $(n=\mid 38,757)$} \\
\hline Underweight & 30.3 \\
\hline Not underweight & 69.7 \\
\hline \multicolumn{2}{|c|}{ Wasting status $(n=\mid 40,255)$} \\
\hline Wasted & 10.4 \\
\hline Not wasted & 89.6 \\
\hline
\end{tabular}

Abbreviation: SSA, sub-Saharan Africa.

were modestly raised in the supplemented children. Effect size estimate indicated that the increased morbidity in the supplemented group was only trivial $(d<0.2$; Table 4$)$.

\section{VAS and anemia}

The mean $( \pm \mathrm{SD})$ hemoglobin concentration in VAsupplemented children $(10.6 \pm 2.2 \mathrm{~g} / \mathrm{dL})$ was significantly higher than in non-supplemented children $(10.4 \pm 1.9 \mathrm{~g} / \mathrm{dL}$; $t=12.218, P=0.000)$. Likewise, the prevalence of anemia in supplemented children (56.9\%) was significantly lower than in non-supplemented children (60.5\%). In the multivariate model, VAS was associated with $10.1 \%$ (95\% CI: 7.0-13.1) reduction in the odds of anemia.

\section{Discussion}

The secondary analysis based on the large multicountry data indicates that VAS to preschool children is associated with a trivial increase in the occurrence fever, diarrhea and 


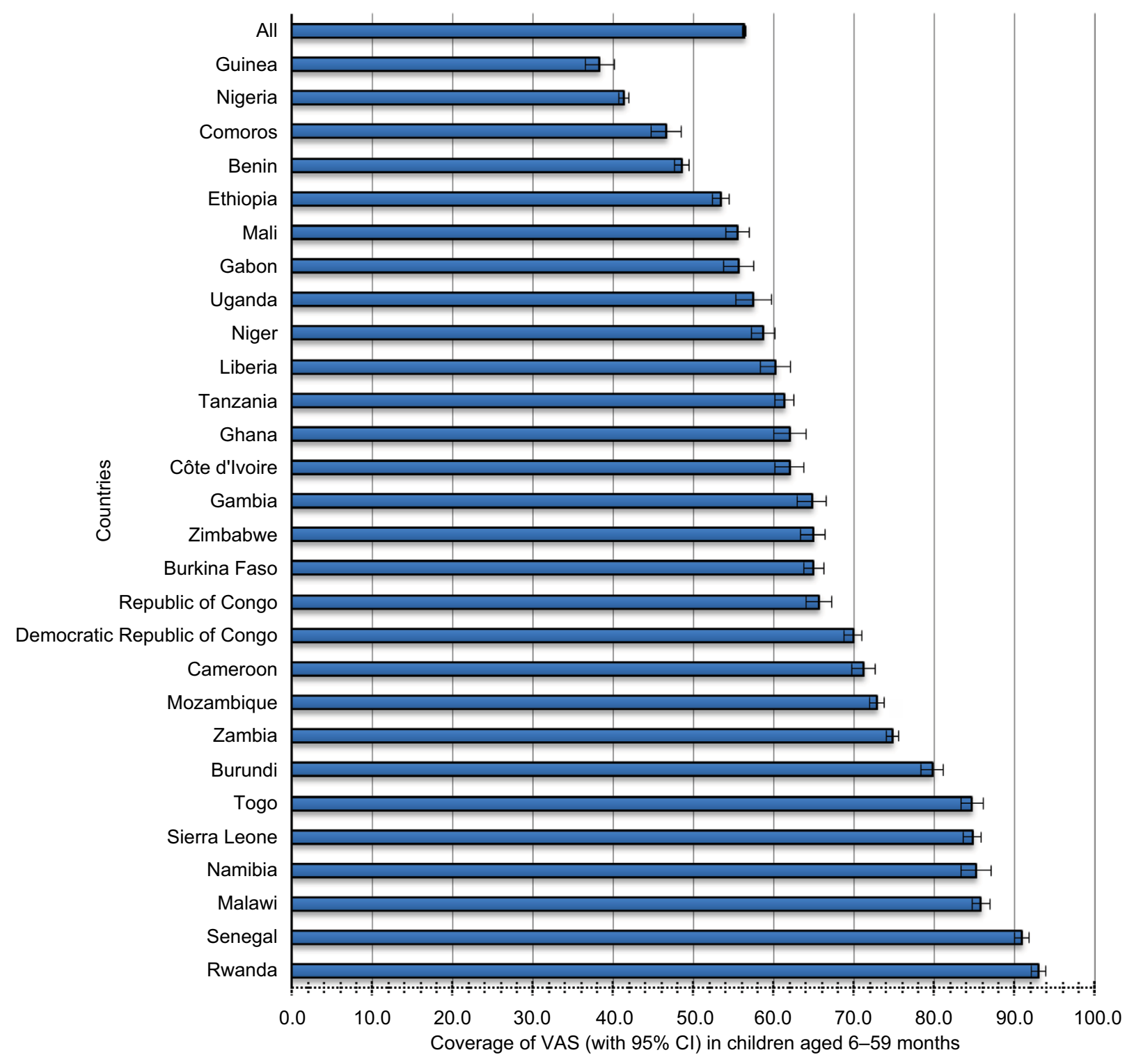

Figure I Coverage of VAS in 28 African countries, 2010-2014.

Abbreviations: $\mathrm{VAS}$, vitamin $\mathrm{A}$ supplementation; $\mathrm{Cl}$, confidence interval.

ARI. Conversely, it is associated with a modest reduction in anemia.

The reported coverage of VAS (56\%) in SSA is below the WHO-recommended $80 \%$ target necessary to achieve child mortality reduction. ${ }^{20}$ The coverage also substantially varies across the subcontinent. Among the 28 countries represented, only seven achieved $\geq 80 \%$ coverage, while four had $<50 \%$ coverage. The figure is also substantially lower than the $73 \%$ coverage reported by UNICEF in 2013 for the entire region of SSA. ${ }^{5}$

Parallel to the association observed in this study, few RCTs indicated that VAS might increase incidence of ARI and diarrhea in pre-school children. In Mexico, VAS increased incidence of diarrhea and cough with fever by 27 and $23 \%$ in children aged 6-15 months. ${ }^{30}$ In Indonesia, VAS increased ARI by $8 \%$ in children aged $6-47$ months and diarrhea by $19 \%$ in children aged $<30$ months. ${ }^{31} \mathrm{~A}$ study conducted in Haiti concluded that children aged 6-83 months supplemented with vitamin A had a higher risk of diarrhea, cough and rapid breathing. ${ }^{32}$ In Tanzania, supplemented children had a higher probability of cough but a smaller risk of diarrhea, but VAS increased diarrhea in well-nourished children. ${ }^{33}$

So far, a convincing mechanism had not been forward to explain how VAS is associated with increased occurrence of ARI and diarrhea. A study hypothesized that high-dose VAS 
for children with adequate vitamin A store may cause a temporary immune malfunction. ${ }^{13}$ Another study concluded that VAS might sometimes downregulate the innate immune due to epigenetic modifications. ${ }^{34,35}$ It has also been hypothesized that the effect of VAS on immunity might be modified by multiple factors, including vitamin $\mathrm{A}$ and anthropometric status of children. ${ }^{36} \mathrm{~A}$ controlled trial in Italy reported that VAS to older population resulted in a significant reduction in the number of CD-3 and CD-4 T-cells. ${ }^{37}$

Systematic reviews as early as 1990s consistently demonstrated that VAS reduces all-cause child mortality by approximately a quarter. ${ }^{8-10,38}$ So how can VAS substantially reduce mortality while having no or negative effect on the incidence

Table 3 Factors associated with utilization of VAS in children aged 6-59 months, 28 SSA countries, 2010-2014

\begin{tabular}{lll}
\hline Variable & $\begin{array}{l}\text { Coverage } \\
\text { of VAS }\end{array}$ & AOR (95\% CI) \\
\hline $\begin{array}{ll}\text { Child's age, months } \\
\text { 6-II }\end{array}$ & 48.6 & $0.66(0.64-0.68)$ \\
$\quad$ I2-59 & 57.9 & $\mathrm{I}^{\mathrm{r}}$ \\
Place of residence & & \\
$\quad$ Urban & 63.9 & $\mathrm{I}^{\mathrm{r}}$ \\
$\quad$ Rural & 53.9 & $0.83(0.8 \mathrm{I}-0.86)$ \\
Household wealth index & & \\
Lowest & 45.5 & $0.42(0.40-0.44)$ \\
Second & $5 \mathrm{I} .4$ & $0.50(0.48-0.52)$ \\
$\quad$ Middle & 57.0 & $0.60(0.57-0.62)$ \\
Fourth & 64.0 & $0.77(0.75-0.80)$ \\
Highest & 70.8 & $\mathrm{I}^{\mathrm{r}}$ \\
Maternal education & & \\
No formal education & 46.7 & $0.45(0.42-0.48)$ \\
Primary education & 63.5 & $0.8 \mathrm{I}(0.76-0.87)$ \\
Secondary education & 66.9 & $0.82(0.77-0.88)$ \\
Tertiary education & 74.9 & $\mathrm{I}^{\mathrm{r}}$ \\
\hline
\end{tabular}

Note: ', reference group.

Abbreviations: VAS, vitamin A supplementation; SSA, sub-Saharan Africa; AOR, adjusted odds ratio; $\mathrm{Cl}$, confidence interval. of the most common causes of childhood morbidity? It has been hypothesized that the protective effect of VAS against mortality might be mediated by a reduction in the severity, rather than the incidence, of illness. ${ }^{10,11}$

The current analysis indicates that VAS might be associated with a modest $10 \%$ reduction in the occurrence of anemia in children. As described earlier, vitamin A appears to be involved in hematogenesis in various ways, including enhancement of growth and differentiation of erythrocyte progenitor cells and mobilization of iron stores. ${ }^{14}$ An analysis of Ethiopian DHS survey concluded that VAS in children aged 6-59 months was associated with a modest but statistically significant increase in hemoglobin and decrease in the risk of anemia. ${ }^{39}$

The current analysis is made based on nationally representative data gathered from 28 countries that contribute for $>75 \%$ of the SSA population. As most of the countries have severe public health problem of VAD, the setting is ideal for assessing the effect of VAS. Conversely, the following limitations of the study should be noted. Although the study attempted to adjust for selected potential confounders, residual confounding from unmeasured or misclassified confounders cannot be ruled out. As the study is observational, it is prone to systematic errors, including selection bias. For instance, the benefits of VAS can be underestimated as VAS campaigns are likely to be implemented more intensively in rural and deficiency-prone areas where the prevalence of childhood illness is high. As exposure to VAS and occurrence of childhood illnesses were determined based on the recall of mothers without any clinical or laboratory investigations, misclassification errors are likely. Furthermore, despite the fact that the effect of VAS on mortality and morbidity can be modified based on a number of variables, including vitamin

Table 4 Association between coverage of VAS and diarrhea, fever and ARI symptoms in 28 African countries, 2010-2014

\begin{tabular}{|c|c|c|c|c|}
\hline VAS & Prevalence of morbidity (\%) & COR $(95 \% \mathrm{Cl})$ & AOR $(95 \% \mathrm{Cl})$ & Effect size $(d ; 95 \% \mathrm{Cl})$ \\
\hline \multicolumn{5}{|l|}{ Fever } \\
\hline Yes & 20.4 & $1.09(1.06-1.12)$ & $1.12(1.09-1.15)$ & \multirow{2}{*}{$0.06(0.05-0.08)$} \\
\hline No & 17.0 & $\mathrm{I}^{\mathrm{r}}$ & $\mathrm{I}^{\mathrm{r}}$ & \\
\hline \multicolumn{5}{|c|}{ Diarrhea } \\
\hline Yes & 14.9 & $1.03(1.00-1.07)$ & $1.09(1.05-1.13)$ & \multirow{2}{*}{$0.05(0.03-0.07)$} \\
\hline No & 13.6 & $\mathrm{I}^{\mathrm{r}}$ & $\mathrm{I}^{\mathrm{r}}$ & \\
\hline \multicolumn{5}{|c|}{ ARI symptoms } \\
\hline Yes & 5.5 & $1.13(1.07-1.19)$ & $1.18(1.16-1.24)$ & \multirow{2}{*}{$0.09(0.06-0.12)$} \\
\hline No & 4.5 & $\mathrm{I}^{\mathrm{r}}$ & $\mathrm{I}^{\mathrm{r}}$ & \\
\hline \multicolumn{5}{|c|}{ Anemia } \\
\hline Yes & 56.9 & $0.83(0.81-0.86)$ & $0.90(0.87-0.93)$ & \multirow{2}{*}{$0.06(0.04-0.08)$} \\
\hline No & 60.5 & $\mathrm{I}^{\mathrm{r}}$ & $\mathrm{I}^{\mathrm{r}}$ & \\
\hline
\end{tabular}

Note: ${ }^{\mathrm{r}}$, reference group.

Abbreviations: VAS, vitamin A supplementation; ARI, acute respiratory infection; COR, crude odds ratio; Cl, confidence interval; $A O R$, adjusted odds ratio. 
A status of the children, ${ }^{36}$ in the current study, interaction assessment has not been made as data on potential effect modifiers were not available.

The large sample size used in the study provided an opportunity to precisely measure small differences that would be unlikely to be detected in ordinary clinical trials and observational studies. Conversely, the study is evidently overpowered. The implication of drawing conclusion from overpowered studies had been discussed in the literature. Large sample size can make the study to detect practically less meaningful differences as $p$-value is sensitive to sample size. ${ }^{40,25}$ In the current study, the statistically significant $p$-values had low effect sizes. This may indicate that the observed association is trivial and unlikely to be of practical significance.

The intension of this article is not to underestimate the benefits of VAS for child well-being and survival. However, the author hopes that it will contribute to the ongoing debate in the literature regarding its effects on childhood morbidity.

\section{Conclusion}

In SSA, the coverage of routine VAS remains low (56\%). Despite the fact that VAS has an established benefit for the reduction in childhood mortality, in this study, it is linked with a trivial increase in the occurrence of common childhood ailments (fever, cough and diarrhea). Conversely, it showed modest contribution toward the reduction of anemia.

\section{Acknowledgment}

The author acknowledges the DHS Program for granting access to the data.

\section{Disclosure}

The author reports no conflicts of interest in this work.

\section{References}

1. Sommer A, West KPJ. Vitamin A Deficiency: Health, Survival, and Vision. New York: Oxford University Press; 1996.

2. Villamor E, Fawzi WW. Effects of vitamin a supplementation on immune responses and correlation with clinical outcomes. Clin Microbiol Rev. 2005;18(3):446-464

3. Stephensen CB. Vitamin A, infection, and immune function. Annu Rev Nutr. 2001;21:167-192.

4. World Health Organization. Global Prevalence of Vitamin A Deficiency in Populations at Risk 1995-2005: WHO Global Database on Vitamin A Deficiency. Geneva: World Health Organization; 2009.

5. UNICEF [webpage on the Internet]. Vitamin A Deficiency: Child health Events Enable Many Countries in West and Central Africa to Achieve Over 80\% Coverage of Vitamin A Supplementation; 2013. Available from: http://data.unicef.org/nutrition/vitamin-a.html. Accessed April 13,2016
6. Mayo-Wilson E, Imdad A, Herzer K, Yakoob MY, Bhutta ZA. Vitamin A supplements for preventing mortality, illness, and blindness in children aged under 5: systematic review and meta-analysis. $B M J$. 2011;343:d5094.

7. Imdad A, Herzer K, Mayo-Wilson E, Yakoob MY, Bhutta ZA. Vitamin A supplementation for preventing morbidity and mortality in children from 6 months to 5 years of age. Cochrane Database Syst Rev. 2010;12:CD008524.

8. Fawzi WW, Chalmers TC, Herrera MG, Mosteller F. Vitamin A supplementation and child mortality. A meta-analysis. JAMA. 1993;269(7):898-903.

9. Imdad A, Yakoob MY, Sudfeld C, Haider BA, Black RE, Bhutta ZA. Impact of vitamin A supplementation on infant and childhood mortality. BMC Public Health. 2011;11(suppl 3):S20.

10. Beaton G, Martorell R, Aronson K, et al. Effectiveness of Vitamin A Supplementation in the Control of Young Child Morbidity and Mortality in Developing Countries: Nutrition Policy Discussion Paper No. 13; 1993.

11. Villamor E, Fawzi WW. Vitamin A supplementation: implications for morbidity and mortality in children. J Infect Dis. 2000;182(suppl 1):S122-S133.

12. Grotto I, Mimouni M, Gdalevich M, Mimouni D. Vitamin A supplementation and childhood morbidity from diarrhea and respiratory infections: a meta-analysis. J Pediatr. 2003;142(3):297-304.

13. Chen H, Zhuo Q, Yuan W, Wang J, Wu T. Vitamin A for preventing acute lower respiratory tract infections in children up to seven years of age. Cochrane Database Syst Rev. 2008;1:CD006090.

14. Semba RD, Bloem MW. The anemia of vitamin A deficiency: epidemiology and pathogenesis. Eur J Clin Nutr. 2002;56(4):271-281.

15. van den Broek N. Anaemia and micronutrient deficiencies. Br Med Bull. 2003;67:149-160.

16. Zimmermann MB, Biebinger R, Rohner F, et al. Vitamin A supplementation in children with poor vitamin $\mathrm{A}$ and iron status increases erythropoietin and hemoglobin concentrations without changing total body iron. Am J Clin Nutr. 2006;84(3):580-586.

17. West KP, Gernand AD, Sommer A. Vitamin A in nutritional anemia. In: Zimmermann MB, editor. Nutritional Anemia. Zurich: SIGHT and LIFE Press; 2007.

18. The World Bank [webpage on the Internet]. Data: Sub-Saharan Africa (Developing Only); 2015. Available from: http://data.worldbank.org/ region/SSA. Accessed April 16, 2016.

19. World Health Organization. Vitamin A Supplementation for Infants and Children 6-59 Months of Age: Guideline. Geneva: WHO Press; 2011.

20. Ross DA. Recommendations for vitamin A supplementation. J Nutr. 2002;132(9 suppl):2902-2906.

21. Horton S, Begin F, Greig A, Lakshman A. Best Practice Paper: Micronutrient Supplements for Child Survival (Vitamin A and Zinc). Copenhagen: Copenhagen Copenhagen Consensus Center; 2008.

22. International ICF. Demographic and Health Surveys Methodology. Calverton, MD: ICF International; 2012.

23. Nestel P. Adjusting Hemoglobin Values in Program Surveys. Washington, DC: International Nutritional Anemia Consultative Group (INACG); 2002.

24. Kaplan RM, Chambers DA, Glasgow RE. Big data and large sample size: a cautionary note on the potential for bias. Clin Transl Sci. 2014;7(4):342-346.

25. Sullivan GM, Feinn R. Using effect size - or why the P value is not enough. J Grad Med Educ. 2012;4(3):279-282.

26. DeCoster J [homepage on the Internet]. Effect Size Calculator; 2012. Available from: www.stat-help.com. Accessed April 16, 2016.

27. Cohen J. Statistical Power Analysis for the Behavioral Sciences. Hillsdale, NJ: Erlbaum; 1988.

28. WHO, UNICEF [webpage on the Internet]. Improved and Unimproved Water Sources and Sanitation Facilities. Available from: http://www. wssinfo.org/definitions-methods/watsan-categories. Accessed April 16 , 2016. 
29. The DHS Program [webpage on the Internet]. Demographic and Health Surveys: Data. Available from: http://dhsprogram.com/Data/. Accessed May 7, 2017.

30. Long KZ, Montoya Y, Hertzmark E, Santos JI, Rosado JL. A doubleblind, randomized, clinical trial of the effect of vitamin A and zinc supplementation on diarrheal disease and respiratory tract infections in children in Mexico City, Mexico. Am J Clin Nutr. 2006;83(3):693-700.

31. Dibley MJ, Sadjimin T, Kjolhede CL, Moulton LH. Vitamin A supplementation fails to reduce incidence of acute respiratory illness and diarrhea in preschool-age Indonesian children. $J$ Nutr. 1996;126(2):434-442.

32. Stansfield SK, Pierre-Louis M, Lerebours G, Augustin A. Vitamin A supplementation and increased prevalence of childhood diarrhoea and acute respiratory infections. Lancet. 1993;342(8871):578-582.

33. Fawzi WW, Mbise R, Spiegelman D, Fataki M, Hertzmark E, Ndossi G. Vitamin A supplements and diarrheal and respiratory tract infections among children in Dar es Salaam, Tanzania. J Pediatr. 2000;137(5):660-667.
34. Arts RJ, Blok BA, van Crevel R, et al. Vitamin A induces inhibitory histone methylation modifications and down-regulates trained immunity in human monocytes. J Leukoc Biol. 2015;98(1):129-136.

35. Arts RJ, Benn CS. Vitamin A induces long-term epigenetic modification in the innate immune system. Sight Life. 2015;29(2):17-20.

36. Griffiths JK. The vitamin A paradox. J Pediatr. 2000;137(5):604-607.

37. Fortes C, Forastiere F, Agabiti N, et al. The effect of zinc and vitamin A supplementation on immune response in an older population. $J \mathrm{Am}$ Geriatr Soc. 1998;46(1):19-26.

38. Glasziou PP, Mackerras DE. Vitamin A supplementation in infectious diseases: a meta-analysis. BMJ. 1993;306(6874):366-370.

39. Gebremedhin S. Effect of a single high dose vitamin A supplementation on the hemoglobin status of children aged 6-59 months: propensity score matched retrospective cohort study based on the data of Ethiopian Demographic and Health Survey 2011. BMC Pediatr. 2014;14:79.

40. Coffey CS, Muller KE. Properties of internal pilots with the univariate approach to repeated measures. Stat Med. 2003;22(15):2469-2485.
Nutrition and Dietary Supplements

\section{Publish your work in this journal}

Nutrition and Dietary Supplements is an international, peer-reviewed, open access journal focusing on research into nutritional requirements in health and disease, impact on metabolism and the identification and optimal use of dietary strategies and supplements necessary for normal growth and development. The journal welcomes submitted papers covering original research, basic science,

\section{Dovepress}

clinical \& epidemiological studies, reviews and evaluations, guidelines, expert opinion and commentary, case reports and extended reports. The manuscript management system is completely online and includes a very quick and fair peer-review system, which is all easy to use. Visit http://www.dovepress.com/ testimonials.php to read real quotes from published authors. 